\title{
Disección sub-mucosa endoscópica en cáncer gástrico incipiente*
}

\author{
Drs. ANDRÉS DONOSO D. ${ }^{1}$, ALLAN SHARP P. ${ }^{1}$, JOSÉ GELLONA V. ${ }^{1}$, ADOLFO PARRA B. ${ }^{2}$, \\ FERNANDO PIMENTEL M. ${ }^{1}$, ALEX ESCALONA P. ${ }^{1}$, OSVALDO LLANOS L. ${ }^{\text {, }}$
}

\author{
Departamento de Cirugía Digestiva. División de Cirugía. \\ 2 Departamento de Gastroenterología. División de Medicina. \\ Facultad de Medicina. Pontificia Universidad Católica de Chile. \\ Santiago, Chile.
}

\begin{abstract}
\section{Endoscopic sub-mucosal dissection for early gastric cancer}

Introduction: Gastric cancer is the leading cause of mortality in Chile and the survival rates are in direct relation with the stage of the disease. Early gastric cancer is defined as that confined to the mucosa or submucosa, regardless of the presence or absence of regional lymph node metastases and it is characterized for its great prognosis, with a survival rate of more than $90 \%$ at ten years of follow up. Endoscopic submucosal dissection is a technique described and developed in Japan that allows the endoscopic complete en-bloc resection of lesions of more than $2 \mathrm{~cm}$ with tumor cell-negative margins. Method: Databases (Pubmed, EBSCO, Cochrane) were reviewed under the terms "gastric cancer", "early gastric cancer", "endoscopic submucosal dissection". Results: The results of published series demonstrate survival rates comparable to standard surgery with less morbidity and without mortality. The present revision describes the endoscopic submucosal dissection technique in early gastric cancer, its indications, the results, the analysis of the resected pieces, the complications and the follow up of the patients. Conclusion: Endoscopic submucosal dissection is a feasible technique, with excellent oncologic results and low morbidity in selected patients.

Key words: Endoscopic submucosal dissection, early gastric cancer, gastric cancer.

\section{Resumen}

Introducción: El cáncer gástrico es la primera causa de muerte por cáncer en Chile y la sobrevida se encuentra en directa relación con la etapa de la enfermedad. El cáncer gástrico incipiente se define como aquel cáncer limitado a la mucosa o sub-mucosa independiente del compromiso ganglionar y se caracteriza por su excelente pronóstico, con sobrevida mayor a 90\% a 10 años de seguimiento. La disección sub-mucosa endoscópica es una técnica descrita y desarrollada en Japón que permite la resección en bloque y con márgenes negativos de tumores incipientes mayores a $2 \mathrm{~cm}$ por vía endoscópica. Método: Se realizó una búsqueda en las bases de datos (Pubmed, EBSCO, Cochrane) bajo las palabras clave: "gastric cáncer", "early gastric

*Recibido el 27 de agosto de 2012 y aceptado para publicación el 14 de septiembre de 2012.

Los autores no refieren conflictos de interés.

Correspondencia: Dr. Andrés Donoso D.

Marcoleta 372, Santiago, Chile.

aidonoso@gmail.com 
cáncer", "endoscopic submucosal dissection". Resultados: Los resultados de grandes series demuestran una sobrevida comparable a la cirugía clásica, con menor morbilidad y sin mortalidad asociada. La presente revisión describe la técnica de disección sub-mucosa endoscópica en cáncer gástrico incipiente, sus indicaciones, los resultados, el análisis de las piezas resecadas, las complicaciones y el modo de seguimiento de los pacientes. Conclusión: La disección sub-mucosa endoscópica es una alternativa válida, con buenos resultados a largo plazo en pacientes debidamente seleccionados.

Palabras clave: Cáncer gástrico incipiente, cáncer gástrico, disección endoscópica submucosa.

\section{Relevancia del cáncer gástrico}

Chile, junto a Japón, Costa Rica y Singapur, presenta la tasa más alta de cáncer gástrico a nivel mundial ${ }^{1}$; en nuestro país, el cáncer gástrico constituye la primera causa de muerte oncológica. Si bien la mortalidad por esta enfermedad ha experimentado un descenso progresivo desde la década de 1970, continúa siendo un importante problema de salud pública, con una mortalidad aproximada de 20 por 100.000 habitantes y una estimación de 3.000 muertes anuales ${ }^{2}$.

La sobrevida por cáncer gástrico se encuentra en directa relación con la etapa de la enfermedad, los pacientes en etapa I tienen una sobrevida a 5 años cercana al 90\%, dependiendo del grado de invasión de la pared gástrica y de la presencia o ausencia de metástasis linfáticas, mientras que aquellos pacientes en etapa IV tienen una sobrevida a 5 años cercana al $10 \%$ dependiendo de las distintas series analizadas $^{3,4}$. Lamentablemente, cerca de un $40 \%$ de los pacientes se presenta con enfermedad diseminada al momento del diagnóstico 5 .

\section{Cáncer gástrico incipiente}

\section{Definición}

Se define cáncer gástrico incipiente como aquel cáncer limitado a la mucosa o a la sub-mucosa del estómago, independiente de la presencia de metástasis linfáticas ${ }^{6}$.

\section{Clasificación}

En 1962, la Sociedad Japonesa de Endoscopia Gastroenterológica propuso la clasificación macroscópica del cáncer gástrico incipiente ${ }^{7}$ (Figura 1). De esta manera, se dividen las lesiones en aquellas solevantadas (Tipo 0I), superficiales (Tipo 0II) y excavadas (Tipo 0III). A su vez, las lesiones superficiales o Tipo 0II se subdividen en lesiones levemente elevadas (Tipo 0IIa), lesiones planas (Tipo 0IIb) y lesiones levemente deprimidas (Tipo 0IIc) ${ }^{8}$.

\section{Pronóstico}

El pronóstico del cáncer gástrico incipiente es excelente. Este depende principalmente de la pro- fundidad en la invasión de la pared gástrica $(\mathrm{T})$ y de la presencia de metástasis linfáticas $(\mathrm{N})$. La mayoría de las series publicadas dan cuenta de una sobrevida a 5 años superior a $90 \%$, destacando las series Japonesas por su importante casuística y por la sobrevida alcanzada, que en algunas series supera el $90 \%$ a 10 años de seguimiento ${ }^{9}$. En Chile existen trabajos que muestran una sobrevida a 5 y a 10 años de seguimiento cercana a $95 \%{ }^{10,11}$. Por lo tanto, el cáncer gástrico incipiente tiene una gran importancia, ya que representa una posibilidad real de curación para los pacientes.

\section{Incidencia de metástasis linfáticas:}

Existen diversos trabajos de anatomía patológica que han estudiado la probabilidad de metástasis linfáticas en pacientes con cáncer gástrico incipiente. Aquellos pacientes con compromiso exclusivo de la mucosa (T1a) tienen una probabilidad de metástasis linfáticas cercana a 3\%, mientras que esta aumenta hasta un $19 \%$ cuando se compromete la submucosa $(\mathrm{T} 1 \mathrm{~b})^{12-14}$.

\section{Indicaciones de resección endoscópica en cáncer gástrico incipiente}

La resección endoscópica está indicada en aquellos pacientes sin riesgo de metástasis linfáticas.

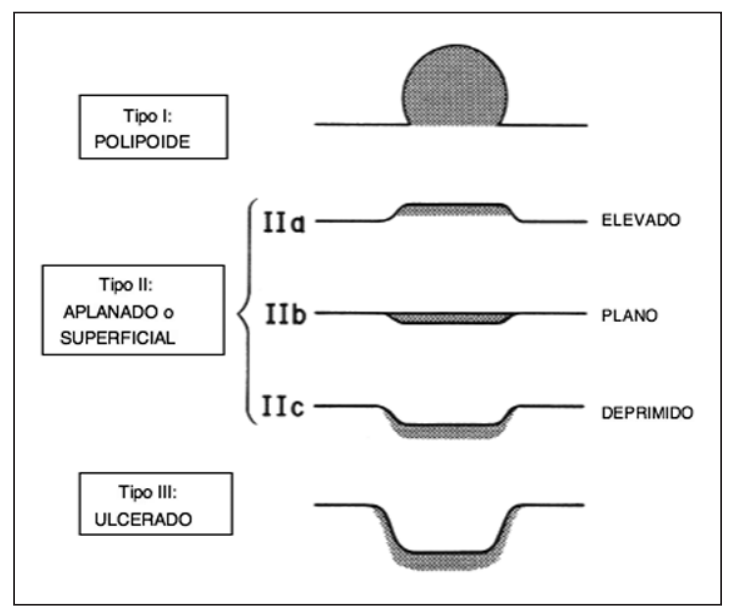

Figura 1. Clasificación del cáncer gástrico incipiente. 
Existen criterios para determinar qué pacientes no presentan riesgo:

Criterios clásicos:

Los criterios "clásicos" incluidos en las Guías Japonesas como indicación de resección endoscópica $\operatorname{son}^{15}$ :

1) Tumor menor a $2 \mathrm{~cm}$.

2) No ulcerado.

3) Intramucoso (T1a o Tis).

4) Histológicamente bien diferenciado.

\section{Criterios extendidos}

En 2000 Gotoda y cols, analizaron la incidencia de metástasis linfáticas en más de 5.000 pacientes con diagnóstico de cáncer gástrico incipiente sometidos a gastrectomía más linfadenectomía D2 ${ }^{16}$. Los hallazgos más importantes de este estudio fueron los siguientes:

1) Ninguno de los 1.230 pacientes con tumores que comprometían exclusivamente la mucosa, histológicamente bien diferenciados y menores a $3 \mathrm{~cm}$ de diámetro presentaba metástasis linfáticas.

2) Ninguno de los 929 pacientes con tumores que comprometían exclusivamente la mucosa, histológicamente bien diferenciados y sin úlcera, presentaba metástasis linfáticas, independiente del diámetro de la lesión.

3) Aquellos pacientes con tumores indiferenciados, no presentaban metástasis linfáticas cuando el tumor comprometía exclusivamente la mucosa, medía menos de $2 \mathrm{~cm}$ de diámetro y la lesión no presentaba ulceración (141 pacientes).

4) En aquellos pacientes en los que el tumor traspasa la mucosa e invade la sub-mucosa, ninguno de los 145 pacientes con compromiso exclusivo de la primera capa de la submucosa (SM1), sin invasión linfo-vascular y con un diámetro menor a $3 \mathrm{~cm}$ presentó metástasis linfáticas.

La importancia de este trabajo, es el gran número de pacientes estudiados, lo cual permitió establecer nuevos criterios, llamados "criterios extendidos" (Tabla 1) para identificar aquellos casos en los que la probabilidad de metástasis linfáticas es mínima o inexistente, y que por lo tanto, se beneficiarán de una resección por vía endoscópica.

\section{Tratamiento endoscópico del cáncer gástrico incipiente}

Como hemos visto, existen criterios que permiten seleccionar pacientes con cáncer gástrico incipiente que presentan un riesgo mínimo de metástasis linfáticas y que por lo tanto, se pueden tratar mediante una resección por vía endoscópica. Este enfrentamiento terapéutico se ha llevado a cabo principalmente en Japón, Korea y otros países asiáticos, siendo escasa aún la experiencia en Occidente ${ }^{17}$. Esto se debe a la alta prevalencia de cáncer gástrico en Japón y al gran porcentaje de detección de cáncer gástrico incipiente, el que alcanza en algunos centros de referencia hasta un $70 \%{ }^{18}$. Desde 1990 , el número de resecciones endoscópicas en cáncer gástrico incipiente ha aumentado de forma exponencial en Japón ${ }^{19}$.

\section{Disección sub-mucosa endoscópica (DSE)}

\section{Definición}

Se ha denominado DSE a aquellas técnicas de resección endoscópica en las que se utiliza el corte o disección directa de la capa sub-mucosa que se encuentra debajo del tumor, y que por la naturaleza misma de la técnica, permiten al cirujano-endoscopista tener control sobre los márgenes de la lesión (R0) y lograr la resección tumoral en una sola pieza (resección completa o en bloque) 20-22. $^{2}$.

\section{Descripción de la técnica}

La DSE involucra cuatro pasos principales: marcación de la lesión, inyección de la sub-mucosa, incisión de la mucosa y disección de la sub-mucosa ${ }^{23}$ (Figura 2).

\section{1) Marcación}

En esta etapa se determina en forma precisa los márgenes de la lesión. Para esto se pueden utilizar

Tabla 1. Resumen de las Indicaciones Ampliadas de Disección Submucosa Endoscópica ${ }^{16}$

\begin{tabular}{|lcccccc|}
\hline $\begin{array}{l}\text { Profundidad y } \\
\text { Tamaño }(\mathbf{m m})\end{array}$ & \multicolumn{2}{c}{ Mucoso } & \multicolumn{2}{c}{ Ulcerado } & \multicolumn{2}{c|}{ Submucoso } \\
& $<=\mathbf{2 0}$ & $\mathbf{2 0}$ & $<=\mathbf{3 0}$ & $\mathbf{3 0}$ & $<=\mathbf{3 0}$ & Cualquiera \\
Diferenciado & $\mathrm{RME}$ & $\mathrm{DSE}$ & $\mathrm{DSE}$ & $\mathrm{C}$ & $\mathrm{DSE}$ & $\mathrm{C}$ \\
Indiferenciado & Considerar C & $\mathrm{C}$ & $\mathrm{C}$ & $\mathrm{C}$ & $\mathrm{C}$ & $\mathrm{C}$ \\
\hline
\end{tabular}

DSE: Disección submucosa endoscópica; RME: Resección mucosa endoscópica (indicación clásica); C: Cirugía; SM1: Compromiso de la capa superficial de la submucosa; SM2: Compromiso de capa profunda de la submucosa. 


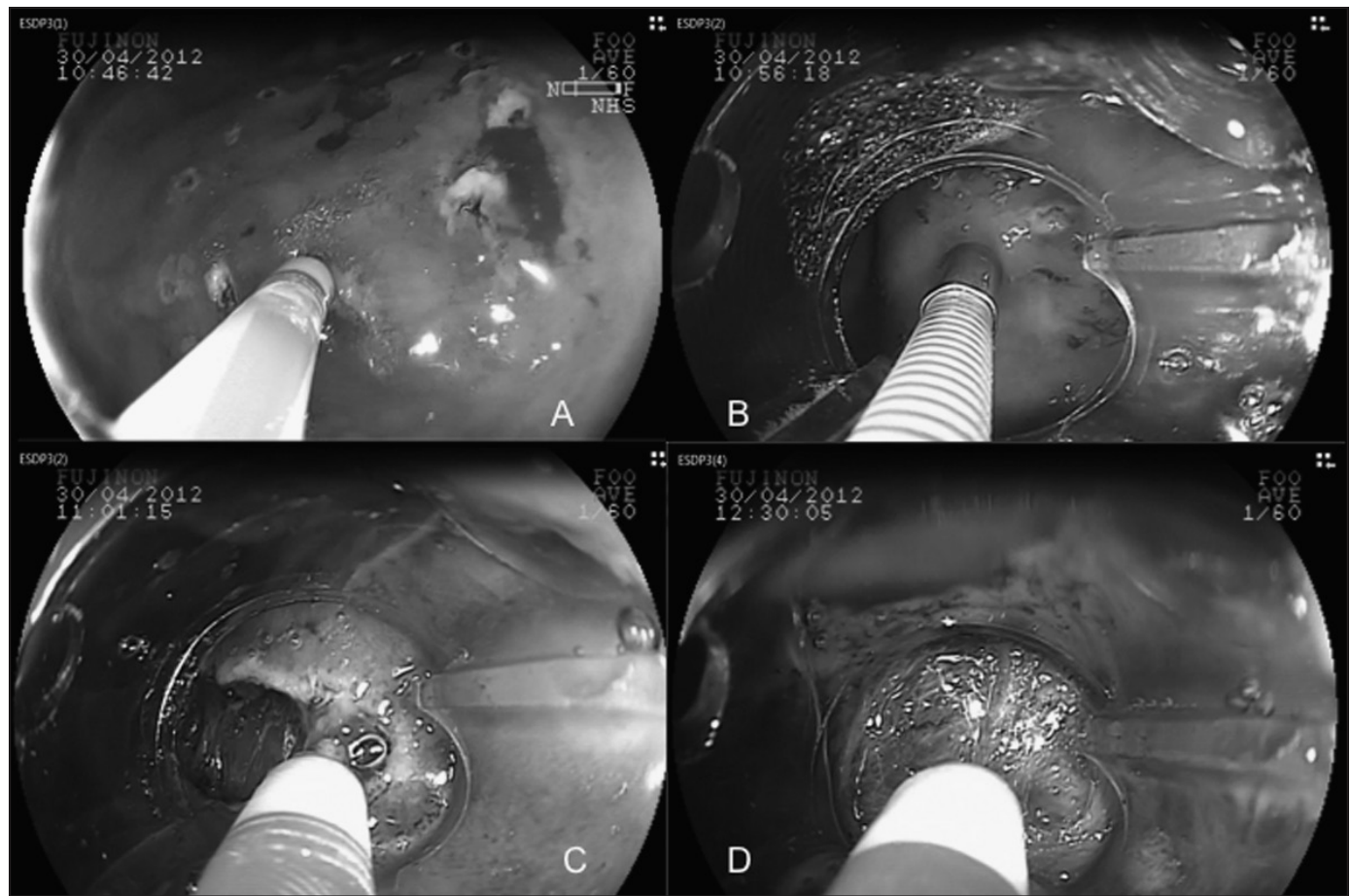

Figura 2. Pasos en la DSE. Marcación de la lesión (A), inyección de la submucosa (B), corte de la mucosa (C) y disección de la submucosa (D).

distintos recursos, como la tinción con Índigo Carmín, la amplificación de imagen o la cromoendoscopia digital. Una vez determinados los márgenes, se realiza una marcación circunferencial por fuera de estos, de manera de incluir toda la lesión en la pieza a resecar (Figura 2-A).

\section{2) Inyección de la sub-mucosa}

Luego de marcar los márgenes se utiliza una aguja de inyecto-terapia con la cual se infiltra la sub-mucosa con una solución que permite elevar la lesión y separar la sub-mucosa de la muscular propia del estómago. Existen distintos tipos de soluciones para este objetivo, siendo actualmente la mezcla de hialuronato de sodio, con índigo carmín y epinefrina la que mejor resultados obtiene ${ }^{24}$. El hialuronato de sodio es una solución viscosa, isotónica y de larga duración. La tinción producida por el índigo carmín permite distinguir más precisamente las fibras de la sub-mucosa y la epinefrina disminuiría el sangrado durante la etapa de corte de la sub-mucosa (Figura 2-B).

\section{3) Incisión de la mucosa}

Posterior a la elevación de la lesión se debe realizar el corte de la mucosa. Esta incisión determina el perímetro de la pieza a resecar, por lo que es de vital importancia que esta sea realizada en forma precisa y siempre por fuera de los puntos de marcación, de manera de asegurar márgenes libres de tumor. El objetivo de la incisión es permitir el acceso a la sub-mucosa previamente elevada durante la etapa de inyección (Figura 2-C).

\section{4) Disección de la sub-mucosa}

En esta etapa se corta y diseca directamente la sub-mucosa por debajo del tumor (Figura 2-D). Para esto existen diversos instrumentos endoscópicos llamados "endo-knives" o cuchillos de disección endoscópica. Estos se pueden clasificar a grandes rasgos en aquellos de punta descubierta o cubierta, siendo los principales ejemplos de cada uno el Flush-Knife (Fujinon) y el IT-Knife (Olympus). Un elemento de gran importancia en esta etapa es el espaciador distal (Figura 2 B, C y D). Este corresponde a una cubierta cónica transparente que se adhiere a la punta del endoscopio y que en algunos casos tiene un menor diámetro en su punta, lo que permite una visualización directa de la sub-mucosa durante la disección sin topar la punta del endoscopio contra ella. Al finalizar esta etapa se reseca completamente el tumor el que se extrae por la boca del paciente con pinzas endoscópicas para posteriormente ser enviado a Anatomía Patológica (Figura 3-A). 


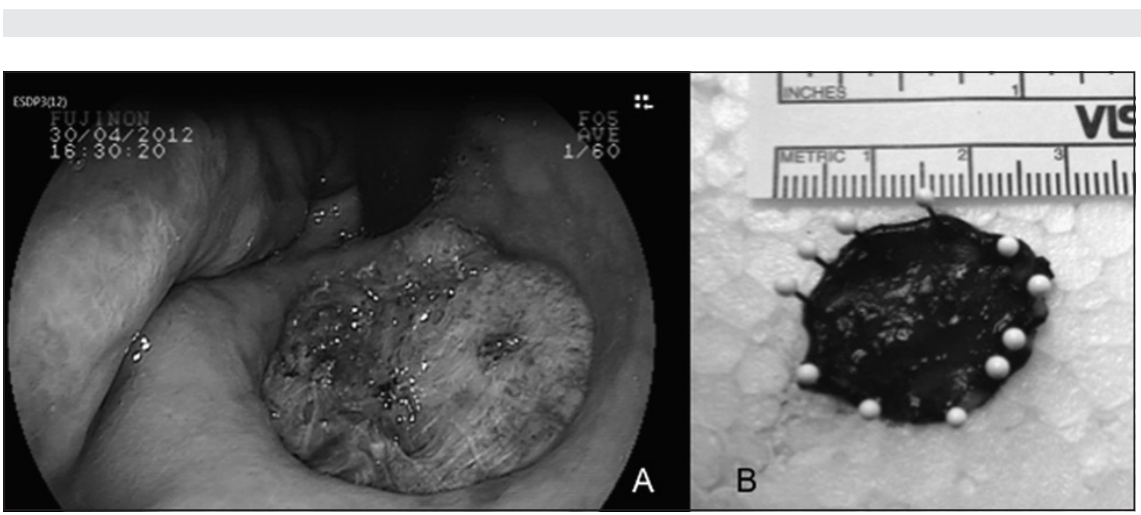

Figura 3. Lecho quirúrgico al extraer la pieza (A), manejo de la pieza previa fijación en formalina (B).

\section{Manejo de la pieza resecada y análisis histo-patológico}

Es importante extender la lesión en una superficie plana afirmando todo su perímetro con alfileres, de esta manera se sumerge la pieza en formalina y se envía para su análisis histopatológico (Figura 3-B). Esta maniobra evita que se retraigan los bordes de la pieza y así poder analizar correctamente los márgenes de resección y medir en forma precisa su diámetro. Posteriormente, se debe procesar la muestra en cortes seriados cada $2 \mathrm{~mm}$.

El informe histo-patológico debe incluir los siguientes puntos ${ }^{24}$ :

1) Tipo histológico y grado de diferenciación.

2) Medidas de la pieza resecada y del tumor (diámetro mayor, menor y profundidad en milímetros).

3) Estado de los márgenes (margen vertical y profundo).

4) Grado de invasión tumoral (si el tumor compromete la sub-mucosa, se debe especificar el grado de compromiso medido en micras desde la muscular de la mucosa).

5) Invasión venosa o linfática.

Sólo una vez que se han determinado en forma precisa los puntos mencionados se puede establecer si el paciente fue sometido o no a una resección curativa.

\section{Resección endoscópica curativa}

Se entiende por resección endoscópica de carácter curativo a aquella en la que se extirpa completamente el tumor, con bordes laterales y profundos no comprometidos, y en la que luego de completar el análisis histo-patológico de la pieza no existen factores de riesgo de metástasis linfáticas (cumple con todos los criterios extendidos).

\section{Resultados}

Existen series con más de 1.000 pacientes tratados mediante esta técnica que reportan un $98 \%$ de resección completa y $93 \%$ de resección R0 sin mor- talidad asociada ${ }^{25}$. Así mismo, la sobrevida reportada a 5 años alcanza un $96,1 \%{ }^{26}$, lo que indica que la DSE en cáncer gástrico incipiente tiene resultados comparables a la cirugía clásica.

\section{Complicaciones}

Las principales complicaciones de esta técnica son la hemorragia y la perforación. Estas tienen una incidencia aproximada de $6 \%$ y $4 \%$ respectivamente. Dentro de los factores de riesgo, el más importante es la ubicación del tumor. Las lesiones ubicadas en el tercio inferior del estómago presentan un mayor riesgo de hemorragia y aquellas ubicadas en el tercio superior presentan mayor riesgo de perforación. El tamaño de la lesión no está relacionado a ninguna de estas complicaciones. La hemorragia suele ser tratada fácilmente por vía endoscópica y en general no presenta mayores problemas para su resolución con los métodos clásicos de hemostasia endoscópica ${ }^{27}$. Así mismo, la perforación de la pared gástrica se trata primariamente por vía endoscópica en la gran mayoría de los casos. Minami y cols, publicaron en 2006 una serie de 2.460 pacientes de los cuales un $4,9 \%$ presentó una perforación gástrica. De estos pacientes, $98 \%$ fueron tratados en forma exitosa por vía endoscópica ${ }^{28}$.

\section{Enfrentamiento terapéutico ante una resección no curativa}

Es importante comentar las alternativas terapéuticas frente a una resección no curativa. Una resección es no curativa cuando el análisis histopatológico de la pieza resecada presenta un margen con compromiso tumoral (lateral o profundo) o cuando presenta factores de riesgo conocidos de metástasis linfáticas (histología indiferenciada, invasión linfo-vascular, ulceración, invasión profunda de la sub-mucosa) ${ }^{29}$. En ocasiones, los pacientes que presentan un margen lateral con compromiso tumoral, con margen profundo libre de tumor y sin factores de riesgo de metástasis linfáticas pueden ser tratados mediante una nueva resección endoscópica. Sin embargo, aquellos pacientes que presentan un margen pro- 
fundo positivo, o que tienen factores de riesgo de metástasis linfáticas, deben ser sometidos a cirugía con disección ganglionar. Esto es de gran importancia, ya que aquellos pacientes operados luego de una resección endoscópica no curativa mantienen un excelente pronóstico ( $97,8 \%$ a 5 años), sin embargo, aquellos pacientes no operados están en riesgo de progresión de la enfermedad, metástasis, y mortalidad por cáncer gástrico ${ }^{30}$.

\section{Seguimiento}

El seguimiento de los pacientes sometidos a una DSE es obviamente de importancia mayor ya que al preservar el estómago la mucosa gástrica se encuentra en riesgo de presentar un nuevo cáncer. Los distintos estudios demuestran la aparición de un nuevo cáncer en $0,82 \%$ de los pacientes tratados por cáncer gástrico incipiente mediante $\mathrm{DSE}^{31}$. El tiempo promedio de detección es de 3 años y los estudios indican que el seguimiento endoscópico anual permite detectar las recurrencias en etapas tempranas y susceptibles de un nuevo tratamiento curativo $^{32}$.

\section{Comentarios finales}

La DSE es actualmente la mejor técnica disponible para la resección endoscópica del cáncer gástrico en etapa incipiente que no presenta riesgo de metástasis linfáticas. Esto es debido a que se tiene control sobre los márgenes de resección laterales y profundos, como así también una visualización directa del tejido sobre el que se está realizando la disección. La DSE permite además resecar lesiones de mayor tamaño, por lo tanto, las indicaciones de resección endoscópica están dadas por criterios oncológicos, es decir, por la probabilidad de metástasis linfáticas y no por limitaciones propias de la técnica.

Como hemos visto, en casos debidamente seleccionados los resultados de sobrevida son comparables a la cirugía clásica y a diferencia de esta, la DSE no presenta mortalidad asociada. Si bien no existen estudios de calidad de vida que comparen cirugía clásica con DSE, el preservar el estómago sugiere claras ventajas para los pacientes y un seguimiento adecuado permite detectar las recurrencias en etapa precoz y susceptible de tratamiento.

La gran mayoría de la experiencia en esta técnica proviene de Japón. Como hemos visto, el porcentaje de detección de cáncer gástrico incipiente alcanza en centros de referencia hasta un $70 \%$, muy por encima de nuestras cifras actuales. En Chile, la gran mayoría de las series reporta un porcentaje de cáncer gástrico incipiente cercano a $10 \%$, sólo en algunos trabajos esta cifra ha llegado a $15 \%$ o $30 \%$ en los mejores $\operatorname{casos}^{5,10,11}$.
Es conocido el programa de tamizaje para la detección de cáncer gástrico en Japón, sin embargo, la gran mayoría de los pacientes a quienes se les diagnostica un cáncer gástrico incipiente en dicho país no provienen del programa de tamizaje ${ }^{33}$, más aún, no existe actualmente evidencia sólida que apoye en forma categórica la implementación de un programa como este en cuanto a disminución de la mortalidad por cáncer gástrico ${ }^{34}$. Sin embargo, dada la relevancia que tiene el cáncer gástrico en nuestro país, no podemos estar ajenos a la importancia de su detección precoz, ni a la adecuada implementación de las distintas técnicas de resección endoscópica.

Consideramos que los esfuerzos en Chile deben estar orientados a mejorar la calidad de la endoscopia. En la década de 1960 la tasa de detección de cáncer gástrico incipiente en Japón era 17\%, aumentando a $30 \%$ en la década de $1970^{35}$ y luego progresivamente hasta las cifras actuales que llegan a $70 \%{ }^{18}$ lo que hace pensar que nuestras cifras son ciertamente mejorables. Actualmente, el desarrollo de la endoscopia permite la detección del cáncer gástrico incipiente en base a sutiles cambios en la disposición de pliegues, vascularización de la mucosa, o cambios en la coloración de la misma. Obviamente, esto exige la implementación de protocolos que permitan un tiempo mínimo de examen, una adecuada preparación del estómago del paciente, una adecuada sedación, un equipo de enfermería y auxiliares técnicos debidamente capacitados y conscientes de la importancia de estos procedimientos. Así mismo, los médicos endoscopistas debemos estar entrenados y capacitados en la correcta evaluación del estómago e idealmente en la implementación de técnicas de magnificación, tinción y cromoendoscopia digital. Como sabemos, la implementación de estos recursos es muchas veces difícil en nuestro medio, pero nuestros esfuerzos deben estar orientados a ello, de manera de poder aumentar el bajo número de lesiones incipientes que actualmente se detectan.

Por último, se debe enfatizar que el tratamiento del cáncer gástrico debe ser multidisciplinario, multimodal e individualizado al paciente. Es importante desarrollar e implementar correctamente la cirugía y la quimio-radioterapia, pero así mismo, debemos estar conscientes de sus limitaciones en el estado del conocimiento actual. La detección del cáncer gástrico en etapa incipiente es probablemente una oportunidad de disminuir la mortalidad por cáncer gástrico en nuestro país.

\section{Referencias}

1. Parkin M, Bray F, Ferlay J, Pisani P. Global Cancer Statistics, 2002 CA Cancer J Clin. 2005;55:74-108. 
2. Serra I, Baez S, Serra J, Calvo A, Decinti E. Evolución epidemiológica reciente del cáncer gástrico en Chile y el mundo. Rev Chil Cir. 1997;129:749-55.

3. Llanos O, Butte JM, Crovari F, Duarte I, Guzmán S. Survival of young patients after gastrectomy for Gastric Cancer. World J Surg. 2006:30;17-20.

4. Burmeister R, Pepper J, Fernández M. Sobrevida a largo plazo del cáncer gástrico. Rev Chil Cir. 1986;38:193-96.

5. Calvo A. Diagnóstico precoz del cáncer gástrico. Rev Med Clin Condes 2011;22:477-84.

6. Japanese Gastric Cancer Association. Japanese classification of gastric carcinoma-2nd English edition. Gastric Cancer 1998;1:10-24.

7. Murakami T. Early Gastric Cancer, Japanese Cancer Asociation. Tokyo: University of Tokyo Press, 1971.

8. Ichikawa H. A modified proposal on the classification for gastric carcinoma. Japanese Joint Committee. Tokyo: Igaku Shoin Co, 1968.

9. Everett SM, Axon ATR. Early gastric cancer in Europe. Gut 1997;41:142-50.

10. Butte JM, Torres J, Viviani P, Duarte I, Crovari F, Guzmán S, y cols. Sobrevida alejada de pacientes operados por cáncer gástrico incipiente. Rev Med Chile 2008;136:1424-30.

11. Ceroni M, García C, Benavides C, Covacevich S, Rubilar P, Cid H, y cols. Seguimiento mayor a 10 años de pacientes operados por cáncer gástrico incipiente. Rev Chil Cir. 2011;63:591-8.

12. Yamao T, Shirao K, Ono H. Risk factors for lymph node metastasis from intramucosal gastric carcinoma. Cancer 1996;77:602-6.

13. Kurihara N, Kubota T, Otani Y. Lymph node metastasis of early gastric cancer with submucosal invasion. Br J Surg 1998;85:835-9.

14. Yasuda K, Shiraishi N, Suematsu T. Rate of Detection of Lymph Node Metatasis Is Correlated with the Depht of Submucosal Invasion in Early Gastric Carcinoma. Cancer 1999;85:2119-23.

15. Nakajima T. Gastric cancer treatment guidelines in Japan. Gastric Cancer 2002;5:1-5.

16. Gotoda T, Yanagisawa A, Sasako M. Incidence of lymph node metastasis from early gastric cancer: estimation with a large number of cases at two large centers. Gastric Cancer 2000;3:219-25.

17. Coda S, Trentino P, Antonellis F. A Western singlecenter experience with endoscopic sub-mucosal dissection for early gastrointestinal cancers. Gastric Cancer 2010;13:258-63.

18. Oshima A. Secondary Prevention: screening methods in high incidence areas. Sugimura T and Sasako M. Gastric Cancer. United States. Oxford University Press. 1997;199-212.

19. Ono H, Kondo H, Gotoda T. Endoscopic mucosal resection for treatment of early gastric cancer. Gut 2001;48:225-9.

20. Park JM, Cho E, Young KH, Kim JM. The effective- ness and safety of endoscopic submucosal dissection compared with endoscopic mucosal resection for early gastric cancer: a systematic review and metaanalysis. Surg Endosc. 2011;25:2666-7.

21. Min BH, Lee JH, Kim JJ. Clinical outcomes of ESD for treating Early Gastric Cancer: Comparisson with EMRP. Digestive and Liver Disease 2009;41:201-9.

22. Gotoda T, Yamamoto H, Soetikno RM. Endoscopic submucosal dissection for early gastric cancer. J Gastroenterol. 2006;41:929-42.

23. Mönkemüller K, Wilcox CM, Muñoz-Navas M (eds): Interventional and Therapeutic Gastrointestinal Endoscopy. Front Gastrointest Res. Basel, Karger 2010; 27:156-68.

24. Fujishiro M, Yahagi N, Kashimura K, Mizushima Y, Oka M, Enomoto S, et al. Comparison of various submucosal injection solutions for maintaining mucosal elevation during endoscopic mucosal resection. Endoscopy 2004;36:579-83.

25. Oda I, Gotoda T, Hamanaka H. Endoscopic submucosal dissection for early gastric cancer: technical feasibility, operation time and complications from a large consecutive series. Digestive Endoscopy 2005;17:54-8.

26. Oda I, Saito D, Tada M. A multicenter study of endoscopic resection for early gastric cancer. Gastric Cancer 2006;9:262-70.

27. Gotoda T, Yamamoto H, Soetikno RM. Endoscopic submucosal dissection of early gastric cancer. J Gastroenterol. 2006;41:929-42.

28. Minami SH, Gotoda T, Ono H. Complete endoscopic closure of gastric perforation induced by endoscopic resection of early gastric cancer using endoclips can prevent surgery. Gastrointest Endosc. 2006;63:596-601.

29. Nagano H, Ohyama S, Fukunaga T. Indications for gastrectomy after incomplete EMR for early gastric cancer. Gastric Cancer 2005;8:149-54.

30. Oda I, Gotoda T, Sasako M. Treatment strategy after non-curative endoscopic resection of early gastric cancer. Br J Surg. 2008;95:1495-500.

31. Park JM, Cho E, Young KH, Kim JM. The effectiveness and safety of endoscopic submucosal dissection compared with endoscopic mucosal resection for early gastric cancer: a systematic review and metanalysis. Surg Endosc. 2011; 25:2666-77.

32. Metachronous gastric cancer after endoscopic resection: how effective is annual endoscopic surveillance? Nakajima T, Oda I, Gotoda T. Gastric Cancer 2006;9:93-8.

33. Suzuki H, Gotoda T, Sasako M. Detection of early gastric cáncer: misunderstanding the role of mass screening. Gastric Cancer 2006:9:315-9.

34. Hamashima C, Shibuya D, Yamazaki H, Inoue K, Fukao A, Saito H, et al. The Japanese Guidelines for Gastric Cancer Screening. Jpn J Clin Oncol. 2008;38:259-67.

35. Ohta H, Noguchi Y, Takagi K, Nishi M, Kajitani T, Kato Y. Early gastric carcinoma with special reference to macroscopic classification. Cancer 1987;60:1099-106. 